\title{
ON GENERAL COHOMOLOGY THEORY
}

\author{
TIBOR RADO
}

Introduction. Due to the somewhat complicated character of the original definition of the Cech cohomology groups (in terms of open coverings and direct limit groups), several alternative approaches have been proposed in the literature for the purpose of achieving better conceptual insight or greater technical convenience, or preferably both. The purpose of this note is to propose still another approach to general cohomology theory. For the sake of economy in presentation, we first state our definition of the cohomology groups $H^{p}(X, A)$, for a pair of topological spaces such that $A \subset X$, and defer comments on motivation and relationship to other approaches to the concluding sections.

Definition of cochain complexes. A cochain complex is a mathematical system $M=\left(C^{p}, \delta^{p}\right)$, where $C^{p}$ denotes, for $p=0, \pm 1$, $\pm 2, \cdots$, an Abelian group and $\delta^{p}: C^{p} \rightarrow C^{p+1}$ is a homomorphism such that $\delta^{p+1} \delta^{p}=0$. The elements $c^{p}$ of $C^{p}$ are the $p$-cochains of $M$. A $p$-cochain $c^{p}$ is termed a $p$-cocycle if $\delta^{p} c^{p}=0$. A $p$-coboundary is a $p$-cochain of the form $c^{p}=\delta^{p-1} c^{p-1}$. One verifies readily the following facts. (i) The $p$-cocycles form a subgroup $Z^{p}$ of $C^{p}$. (ii) The $p$-coboundaries form a subgroup $B^{p}$ of $Z^{p}$. Accordingly, one can define the $p$ th cohomology group $H^{p}$ of $M$ as the factor group $Z^{p} / B^{p}$.

Definition of the relative cohomology groups $H^{p}(X, A)$. Let $(X, A)$ be a pair of topological spaces such that $A \subset X$ and $X \neq 0$ (the case $X=0$ is disregarded to avoid trivialities). We associate with the pair $(X, A)$ a cochain complex $M(X, A)$ in the following manner (for clarity, we break down the construction of $M(X, A)$ into several steps).

(i) For $p \geqq 0$, we define a $p$-function $c_{X}^{p}$ as an integral-valued function $c_{X}^{p}\left(x_{0}, \cdots, x_{p}\right)$ of $p+1$ points of $X$. The additive group of $p$-functions is denoted by $\Phi^{p}(X)$. For $p<0$, we define $\Phi^{p}(X)=0$ (that is, a group consisting of a zero-element alone).

(ii) For $p \geqq 0$, the coboundary homomorphism $\delta_{X}^{p}: \Phi^{p}(X) \rightarrow \Phi^{p+1}(X)$ is defined by the standard formula

$$
\left(\delta_{X}^{p} c_{X}^{p}\right)\left(x_{0}, \cdots, x_{p+1}\right)=\sum_{i=0}^{p+1}(-1)^{i} c_{X}^{p}\left(x_{0}, \cdots, \widehat{x}_{i}, \cdots, x_{p+1}\right) .
$$

Presented to the Society, April 28, 1951; received by the editors April 1, 1952 and, in revised form, July 2, 1952. 
For $p<0, \delta_{X}^{p}$ is the trivial zero-homomorphism. Clearly $\delta_{X}^{p+1} \delta_{X}^{p}=0$.

(iii) For $p \geqq 0$, we define now the group $C^{p}(X, A)$ as consisting of those $p$-functions $c_{X}^{p}$ which vanish locally on $X$ and vanish identically on $A$. Explicitly, $c_{X}^{p} \in C^{p}(X, A)$ if and only if the following two conditions hold. (a) For every $x \in X$, there exists an open set $U=U\left(x, c_{X}^{p}\right)$, containing $x$, such that $c_{X}^{p}\left(x_{0}, \cdots, x_{p}\right)=0$ whenever $x_{0}, \cdots, x_{p} \in U$. (b) $c_{X}^{p}\left(x_{0}, \cdots, x_{p}\right)=0$ whenever $x_{0}, \cdots, x_{p} \in A$.

For $p<0$, we set $C^{p}(X, A)=\Phi^{p}(X)=0$.

(iv) It is immediate that $\delta_{X}^{p} C^{p}(X, A) \subset C^{p+1}(X, A)$. Accordingly, the groups $C^{p}(X, A)$ jointly with the homomorphisms $\delta_{X}^{p}$ constitute a cochain complex $M(X, A)$.

(v) We define, finally, $H^{p}(X, A)$ as the $p$ th cohomology group of this cochain complex $M(X, A)$.

Relationship to alternative approaches. Let us recall that one finds in the literature actually two versions of cohomology theory: the unreduced and the reduced theory. The two theories agree except for $A=0, p=0$; for this case, one has an unreduced and a reduced cohomology group. The relationship of our cohomology theory to the classical reduced theory is described by the following statement: if $X, A$ are both compact, then our group $H^{p+1}(X, A)$ is isomorphic to the pth Cech cohomology group of the pair $X, A$. One way of proving this is to verify that our cohomology groups satisfy (with the indicated shift in dimension) the celebrated eight axioms of Eilenberg and Steenrod, which are known to be categorical for compact pairs. The detailed verification of these eight axioms is a task that the specialist in this field will carry out without any difficulty. Suffice it to say that this verification can be made in a very concise and elegant manner if one is willing to devote some special effort to achieve this purpose. An alternative method consists of comparing our cohomology groups with one of the established versions of the Cech cohomology theory. This comparison turns out to be quite simple if one uses the version due to Wallace which has been studied in detail by Spanier (Ann. of Math. vol. 49 (1948) pp. 407-427). Taking again the reduced theory, the result is now not restricted to compact pairs. Rather, one obtains the following conclusion: If $X$ is any topological space, and $A$ is a closed (perhaps empty) subset of $X$, then our cohomology group $H^{p+1}(X, A)$ is isomorphic to the pth cohomology group of the pair $X, A$ in the sense of Wallace. The experienced reader will readily discover the explicit isomorphism that yields the proof of this statement.

Motivation. The reader will observe that our definition of the groups $H^{p}(X, A)$ employs the process of identification with respect to 
an equivalence relation only at one stage, namely, when cocycles are identified modulo coboundaries. Actually, we arrived at this definition of $H^{p}(X, A)$ while trying to set up the relative cohomology groups subject to the following two conditions. (i) $H^{p}(X, A)$ should be the cohomology group of a cochain complex associated with the pair $(X, A)$. (ii) The use of the process of identification should be minimized. Condition (i) is motivated by the desire to take advantage of the technical convenience of the general theory of cochain complexes, while condition (ii) is concerned with conceptual simplicity.

The OHio State University

\section{EFFECTIVENESS AT THE ORIGIN OF THE SUM SET OF BASIC SETS OF POLYNOMIALS}

RAGY AND BUSHRA H. MAKAR

We have recently investigated the effectiveness [2] of the sum set

$$
\begin{aligned}
\left\{u_{n}(z)\right\}=\frac{1}{\lambda+\mu+\cdots+\gamma}\left[\lambda\left\{p_{n}(z)\right\}+\mu\left\{q_{n}(z)\right\}\right. & +\cdots \\
& \left.+\gamma\left\{t_{n}(z)\right\}\right],
\end{aligned}
$$

$\lambda+\mu+\cdots+\gamma \neq 0$, in a circle in which the constituent simple monic sets $\left\{p_{n}(z)\right\},\left\{q_{n}(z)\right\}, \cdots,\left\{t_{n}(z)\right\}$ are each effective. We have also investigated the order [3] of the set $\left\{u_{n}(z)\right\}$ when the constituent sets are of a given order. It is proposed here to investigate the effectiveness at the origin of the set $\left\{u_{n}(z)\right\}$ when its constituent sets are each effective at the origin.

The set $\left\{p_{n}(z)\right\}$ is said to be effective at the origin [4] when it represents every function regular at the origin in some circle surrounding the origin. If the representations of powers of $z$ by the set $\left\{p_{n}(z)\right\}$ are given by

$$
z^{n}=\sum_{i=0}^{n-1} \pi_{n i}(p) \cdot p_{i}(z)+p_{n}(z)
$$

the necessary and sufficient condition that the set $\left\{p_{n}(z)\right\}$ is effective at the origin is that [4]

Received by the editors May 28, 1952. 